\title{
Semiclassical Husimi functions for spin systems
}

\author{
Marcel Novae* $*$ and Marcus A.M. de Aguiar \\ Instituto de Física "Gleb Wataghin", Universidade Estadual de Campinas, 13083-970 Campinas-SP, Brazil
}

\begin{abstract}
We derive a semiclassical approximation to the Husimi functions of stationary states of spin systems. We rederive the Bohr-Sommerfeld quantization for spin by locating the poles of the corresponding local Green function. The residues correspond to the Husimi functions, which are seen to agree very well with exact calculations.
\end{abstract}

Rigorous derivation of semiclassical approximations in phase space via path integrals [1] for systems with one degree of freedom have recently received considerable attention, both for continuous variables and spin systems. Baranger et al 2], for example, have discussed the canonical coherent state path integral and its semiclassical approximation in some detail, including an initial-value representation and the Green function. The study of semiclassical propagation of wave packets, using complex 3 or nearly real [4] trajectories, for regular and chaotic [5] systems, has developed considerably over the last few years. The spin path integral, and its semiclassical approximation, has found an important application in the study of spin tunnelling and topological effects [6]. Stone et al have derived the spin coherent state semiclassical propagator in detail 7], paying particular attention to the so-called Solari-Kochetov [8] correction. This correction is related to the difference between the average value of the Hamiltonian in coherent states and its Weyl symbol [9], and has a counterpart in the canonical case [2].

To obtain semiclassical approximations for the energy levels $E_{n}$ and stationary states $\langle x \mid n\rangle=\psi_{n}(x)$ of onedimensional bound systems, on the other hand, one normally resorts to the usual Bohr-Sommerfeld (BS) and WKB theories [10]. A coherent state version of these theories, which works in phase space, is also available [2] and produces a BS formula and a semiclassical approximation to the Husimi functions $\mathcal{H}_{n}(z)=|\langle z \mid n\rangle|^{2}$. Recently, Garg and Stone 11] have derived a semiclassical (BS-like) quantization condition for spin systems, including the first quantum corrections (see also 12]). By taking the trace of the semiclassical Green function, they obtained the energy levels as the location of its poles. In the present work we have obtained the semiclassical Husimi functions for spin systems.

The non-normalized spin coherent states are defined by $|z\rangle=\exp \left\{z J_{+}\right\}|j,-j\rangle$, and the semiclassical approximation to the propagator $K=\left\langle z_{f}\left|e^{-i \hat{H} t / \hbar}\right| z_{i}\right\rangle$ is []

$$
K_{s c l}\left(\bar{z}_{f}, z_{i}, t\right)=\left(\frac{i}{\hbar} \frac{e^{i \mathcal{B} / j}}{2 j} \frac{\partial^{2} S}{\partial z_{i} \partial \bar{z}_{f}}\right)^{1 / 2} \exp \left\{\frac{i}{\hbar} \Phi\right\}
$$

where the phase is the classical action plus an extra term

*Electronic address: mnovaes@ifi.unicamp.br known as the Solari-Kochetov (SK) correction:

$$
\Phi=S+\mathcal{I}_{S K}=S+\int_{0}^{t} A\left(t^{\prime}\right) d t^{\prime}
$$

The classical spin action is given by

$$
S=\int_{0}^{t}\left[i \hbar j \frac{\bar{z} \dot{z}-\dot{\bar{z}} z}{1+\bar{z} z}-H(z, \bar{z})\right] d t^{\prime}+\mathcal{B},
$$

where the integral is done along the classical trajectory determined by the Hamilton equations of motion

$$
-i \hbar \dot{\bar{z}}=\frac{1}{g(z, \bar{z})} \frac{\partial H}{\partial z}, \quad i \hbar \dot{z}=\frac{1}{g(z, \bar{z})} \frac{\partial H}{\partial \bar{z}}
$$

and the classical Hamiltonian $H(z, \bar{z})$ is the average value of the quantum Hamiltonian, $H(z, \bar{z})=\langle z|\hat{H}| z\rangle /\langle z \mid z\rangle$. This action obeys the Hamilton-Jacobi relations

$$
\frac{i}{\hbar} \frac{\partial S}{\partial \bar{z}_{f}}=\frac{2 j z(t)}{1+\bar{z}_{f} z(t)}, \quad \frac{i}{\hbar} \frac{\partial S}{\partial z_{i}}=\frac{2 j \bar{z}(0)}{1+\bar{z}(0) z_{i}}, \quad \frac{\partial S}{\partial t}=-H .
$$

The function $g(z, \bar{z})$, which is unity in the canonical case, is given by

$$
g(z, \bar{z})=\frac{\partial^{2}}{\partial z \partial \bar{z}} \ln \langle z \mid z\rangle=\frac{2 j}{(1+z \bar{z})^{2}} .
$$

Note that $\mathcal{B}=-i \hbar j \ln \left[\left(1+\bar{z}_{f} z(t)\right)\left(1+\bar{z}(0) z_{i}\right)\right]$ is a boundary term, that takes into account the fact that in general $\bar{z}$ is not the complex conjugate of $z$ (the discrete time formulation of the path integral indicates that the variables $z$ and $\bar{z}$ must be considered as independent, so we denote the actual complex conjugate of $z$ by $z^{*}$ ). That means that if one defines the usual canonical $(q, p)$ variables according to

$$
\frac{z}{\sqrt{1+z \bar{z}}}=\frac{q+i p}{\sqrt{4 \hbar j}}, \quad \frac{\bar{z}}{\sqrt{1+z \bar{z}}}=\frac{q-i p}{\sqrt{4 \hbar j}}
$$

then $q$ and $p$ will in general be complex numbers.

The semiclassical limit for spin systems consists in letting $\hbar \rightarrow 0$ and $j \rightarrow \infty$, but keeping $\hbar j=1$. If the Hamiltonian if $O(\hbar j)$, then $S$ is $O(\hbar j)$, but the SK correction

$$
A=\frac{\partial}{\partial \bar{z}} \frac{1}{4 g(z, \bar{z})} \frac{\partial H}{\partial z}+\frac{\partial}{\partial z} \frac{1}{4 g(z, \bar{z})} \frac{\partial H}{\partial \bar{z}}
$$


is $O(\hbar)$, and therefore can be considered small. Note that since $\bar{z} \neq z^{*}$ the Hamiltonian $H(z, \bar{z})$, the action and the SK correction can all be complex.

The semiclassical Green function,

$$
G_{s c l}(z, E)=\frac{1}{i \hbar} \int_{0}^{\infty} K_{s c l}(\bar{z}, z, t) e^{i E t / \hbar} d t,
$$

can be calculated by making a stationary exponent approximation to the integral. Note that we are interested only in its diagonal elements. This implies $z_{i}=z$, $\bar{z}_{f}=z^{*}$, but in general $z(t) \neq z$ and $\bar{z}(0) \neq z^{*}$, so that we do not have a real periodic orbit (by real orbit we mean one in which $q$ and $p$ are real). The stationary time $t_{0}$ is determined by the condition

$$
\left.\frac{d(\Phi+E t)}{d t}\right|_{t_{0}}=\left.\frac{\partial S}{\partial t}\right|_{t_{0}}+\left.\frac{\partial \mathcal{I}_{S K}}{\partial t}\right|_{t_{0}}+E=0 .
$$

As usual in semiclassical calculations, we do not consider derivatives of $A$, because including such terms would be inconsistent with the gaussian approximation involved in the derivation of (11). Therefore Eq.(10) can be also written as

$$
E-\mathcal{E}\left(z, t_{0}\right)+A\left(z, t_{0}\right)=0,
$$

where

$$
\mathcal{E}\left(z, t_{0}\right)=-\left.\frac{\partial S}{\partial t}\right|_{t_{0}}
$$

is the energy of the classical trajectory, not to be confused with $E$, the argument of the Green function. In order to proceed with the integration, we need to expand the exponent to second order in time. We define

$$
\left.\frac{\partial^{2} S}{\partial t^{2}}\right|_{t_{0}}:=\alpha\left(z, t_{0}\right)
$$

and neglect the second derivative of $I_{S K}$, in order to obtain

$$
G(z, E)=\frac{1}{i \hbar}\left(-\frac{\pi e^{i \mathcal{B} / \hbar j}}{\alpha j} \frac{\partial^{2} S}{\partial z_{i} \partial \bar{z}_{f}}\right)_{t_{0}}^{1 / 2} \exp \left\{\frac{i}{\hbar} \varphi\right\}
$$

where

$$
\varphi=S\left(t_{0}\right)+\mathcal{I}_{S K}\left(t_{0}\right)+E t_{0} .
$$

We can find a more convenient way of expressing $\alpha$ in order to transform the prefactor. The form (see [2])

$$
\alpha=-\dot{z} \dot{\bar{z}} \frac{\partial^{2} S}{\partial \bar{z}_{f} \partial z_{i}}
$$

leads to

$$
G(z, E)=\frac{1}{i \hbar}\left(\frac{\pi e^{i \mathcal{B} / \hbar j}}{\dot{z} \dot{\bar{z}} j}\right)^{1 / 2} \exp \left\{\frac{i}{\hbar} \varphi\right\} .
$$

Even with this simplification it is hard to find the poles of $G(z, E)$. Garg and Stone 11] have done this by calculating its trace under another stationary phase approximation, which leads to $z(t)=z, \bar{z}(0)=z^{*}$, and thus to real periodic orbits. We take a different route, that will allow us to obtain not only the energy levels but also the Husimi distributions. Even though the classical orbits involved in the calculation of (1) and (9) are complex, we argue that the largest contributions to the function $G(z, E)$ (and not only to its trace) must come from the vicinity of the real periodic orbit through $z$, and its repetitions. The accuracy of the final results support this idea.

Let us denote the period of the orbit through $z$ by $T(z)$ and expand the stationary time $t_{0}$ as

$$
t_{0} \simeq n T+T_{0},
$$

where $n$ counts the repetitions of the real periodic orbit and $T_{0}$, assumed small, has to be determined. Expanding the stationary exponent condition (10) we find

$$
T_{0}=-\frac{E-\mathcal{E}+A}{\alpha^{(n)}},
$$

where

$$
\alpha^{(n)}:=\alpha(z, n T)=-\left.\frac{\partial \mathcal{E}}{\partial t}\right|_{n T}=\left.\frac{\partial^{2} S}{\partial t^{2}}\right|_{n T} .
$$
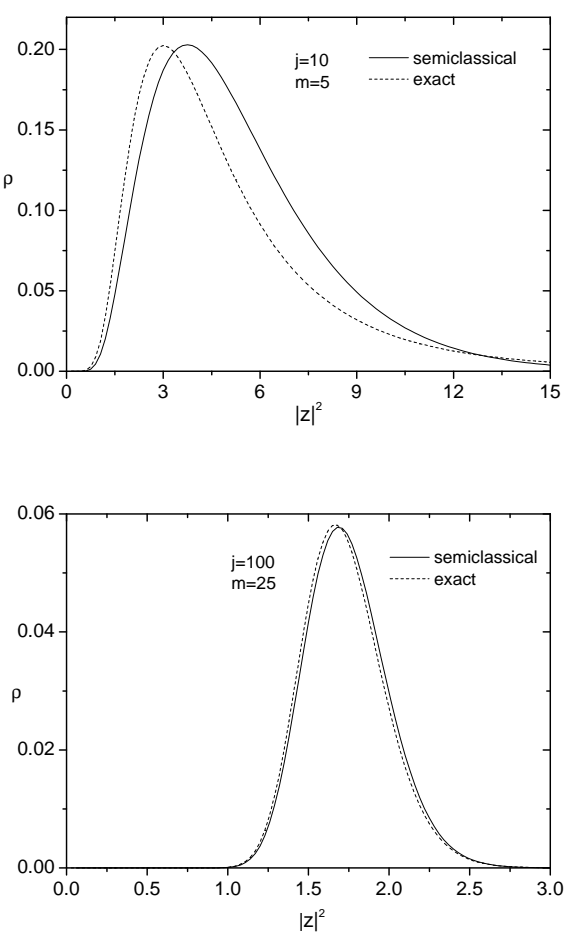

FIG. 1: Exact (top) and semiclassical (bottom) Husimi distributions for the simple Hamiltonian $H=\omega \hbar J_{z}$. 
Now we must expand $\varphi$ to second order in $T_{0}$. Note that $S(n T)=n \mathcal{S}-n \mathcal{E} T+\mathcal{B}(n T)$, where

$$
\mathcal{S}=i \hbar j \int_{0}^{T} \frac{\bar{z} \dot{z}-\dot{\bar{z}} z}{1+\bar{z} z} d t
$$

Note also that $\mathcal{I}_{S K}(n T)=n \mathcal{I}_{S K}(T)$. It can also be shown 2] that

$$
\frac{1}{\alpha^{(n)}}=\frac{1}{i \hbar g(z, \bar{z})|\dot{z}|^{2}}-n \frac{d^{2} \mathcal{S}}{d \mathcal{E}^{2}} .
$$

After $n$ repetitions of a periodic orbit the prefactor acquires a phase of $(-1)^{n}$. Therefore, the result of this expansion is

$$
\begin{aligned}
\varphi & \simeq n\left[\mathcal{S}-\mathcal{E} T+E T+\mathcal{I}_{S K}-\pi \hbar\right] \\
& +\mathcal{B}(n T)-\mathcal{E} T_{0}+\frac{\alpha^{(n)}}{2} T_{0}^{2}+E T_{0}+A T_{0}
\end{aligned}
$$

If we add and subtract $n A T$, define $x=E-\mathcal{E}+A$ and use $T=d \mathcal{S} / d \mathcal{E}$ together with equation (22) we obtain

$$
\begin{aligned}
\varphi & \simeq n\left[\mathcal{S}+\mathcal{I}_{S K}-\pi \hbar+\frac{d \mathcal{S}}{d \mathcal{E}} x-\frac{d \mathcal{S}}{d \mathcal{E}} A+\frac{1}{2} \frac{d^{2} \mathcal{S}}{d \mathcal{E}^{2}} x^{2}\right] \\
& -\frac{1}{g(z, \bar{z})} \frac{x^{2}}{2 i \hbar|\dot{z}|^{2}}+\mathcal{B}(n T) .
\end{aligned}
$$
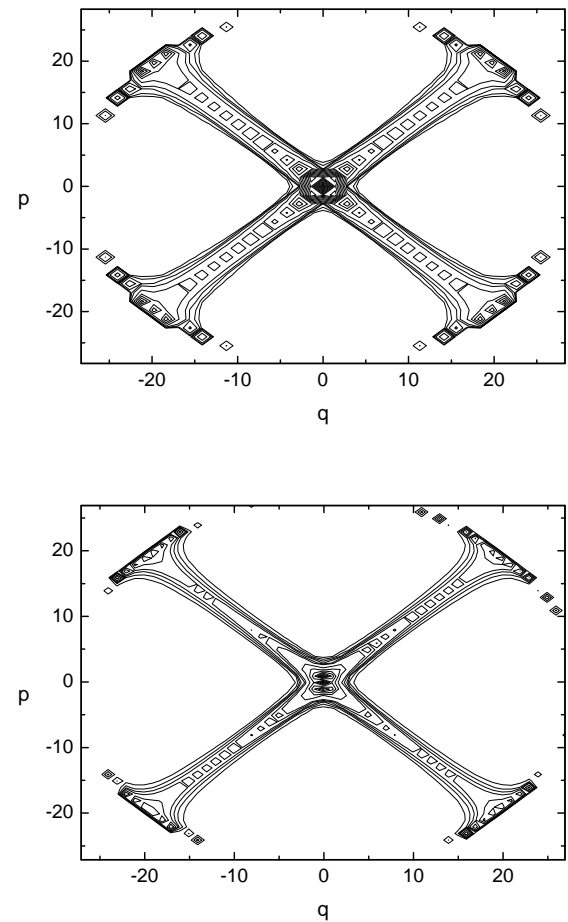

FIG. 2: Exact (top) and semiclassical (bottom) Husimi distributions for the 200th state of the LMG model, with $j=200$, $\omega=1$ and $\hbar \alpha=1000$.
We recognize inside the brackets the expression for the expansion of $\mathcal{S}(E+A)$ around $\mathcal{E}$. Since $A \sim O(\hbar)$ we further expand

$$
\mathcal{S}(E+A) \simeq \mathcal{S}(E)+\frac{d \mathcal{S}}{d \mathcal{E}} A
$$

and we end up with

$$
\varphi \simeq n\left[\mathcal{S}(E)+\mathcal{I}_{S K}(E)-\pi \hbar\right]-\frac{1}{g(z, \bar{z})} \frac{x^{2}}{2 i \hbar|\dot{z}|^{2}}+\mathcal{B}(n T)
$$

Summing over $n$ we obtain

$$
\begin{aligned}
G(z, E) & =\frac{\sqrt{\pi}\left(1+|z|^{2}\right)^{2 j+1}}{i \hbar j|\dot{z}|} \frac{e^{i\left[\mathcal{S}+\mathcal{I}_{S K}-\pi \hbar\right] / \hbar}}{1-e^{i\left[\mathcal{S}+\mathcal{I}_{S K}-\pi \hbar\right] / \hbar}} \\
& \times \exp \left\{-\frac{1}{g(z, \bar{z})} \frac{(E-\mathcal{E}+A)^{2}}{2 \hbar^{2}|\dot{z}|^{2}}\right\}
\end{aligned}
$$

where we have used $\mathcal{B}(n T)=-2 i \hbar j \ln \left(1+|z|^{2}\right)$. The poles of this function are determined by the condition

$$
\left(\mathcal{S}+\mathcal{I}_{S K}\right)\left(E_{n}\right)=(2 n+1) \pi \hbar
$$

which is exactly the Bohr-Sommerfeld quantization rule obtained recently in 11]. The residues at each pole give
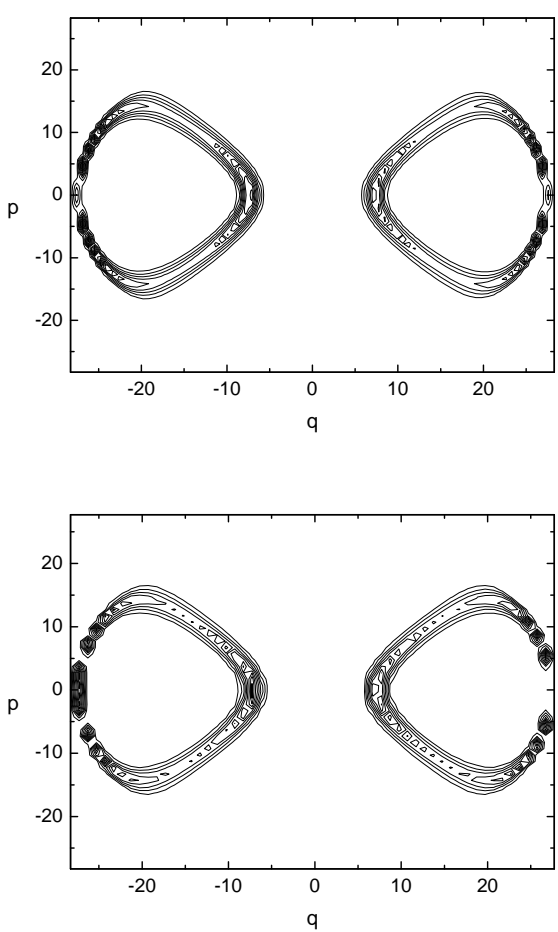

FIG. 3: Exact (top) and semiclassical (bottom) Husimi distributions for the 270th state of the LMG model. The parameters are the same as in the previous figure. 
the Husimi functions

$$
\begin{aligned}
\mathcal{H}_{n}(z) & =\frac{\sqrt{\pi}}{j} \frac{1+|z|^{2}}{|\dot{z}|} \frac{1}{\left[T\left(E_{n}\right)+\left.\left(d \mathcal{I}_{S K} / d \mathcal{E}\right)\right|_{E_{n}}\right]} \\
& \times \exp \left\{-\frac{1}{g(z, \bar{z})} \frac{\left(E_{n}-\mathcal{E}(z)+A(z)\right)^{2}}{2 \hbar^{2}|\dot{z}|^{2}}\right\} .
\end{aligned}
$$

Here we have multiplied by the coherent states normalization factor $\left(1+|z|^{2}\right)^{-2 j}$. These functions are our main result. They are in general not normalized, and have a strong resemblance with the canonical semiclassical Husimi functions presented in 2].

As a first example, we calculate the Husimi function for the simple case $\hat{H}=\hbar \omega J_{z}$. The Hamilton equations can easily be solved and give $z(t)=e^{-i \omega t} z_{i}$ and $\bar{z}(t)=$ $e^{i \omega(t-T)} \bar{z}_{f}$, which implies $|\dot{z}|=\omega|z|$. The SK correction is also very simple, with $A(z)=\hbar \omega / 2$ and $d \mathcal{I}_{S K} / d \mathcal{E}=0$. The final result is

$\mathcal{H}_{m}(r)=\frac{1+r}{2 j \sqrt{\pi r}} \exp \left\{-\frac{(j(1-r)+(1+r)(m+1 / 2))^{2}}{4 j r}\right\}$,

where $m$ goes from $-j$ to $j$ and $r=|z|^{2}$. When properly normalized, this approximates the exact distribution,

$$
\mathcal{H}_{m}^{E}(r)=\frac{(2 j) !}{(j+m) !(j-m) !} \frac{r^{j+m}}{(1+r)^{2 j}},
$$

quite well for large values of $j$, as we can see in Fig 1.

Now let us turn our attention to a less trivial system. In [11] the authors have shown that the semiclassical quantization condition (28) works very well for the
Lipkin-Meshkov-Glick (LMG) model

$$
\hat{H}=\hbar \omega J_{z}+\alpha \hbar^{2}\left[J_{x}^{2}-J_{y}^{2}\right]
$$

already at moderate values of $j$. We now consider the accuracy of the semiclassical approximation (29) for its stationary states. For small values of $\alpha$ the results are very similar to the previous case, so we consider only $j=200, \omega=1$ and $\hbar \alpha=1000$. In order to display the results, we use the canonical coordinates $(q, p)$ given in (7), in terms of which the phase space is compact, $q^{2}+p^{2} \leq 4 \hbar j$. We show the exact and the semiclassical (normalized) Husimi functions for two different states in Fig 2 and Fig 3. The agreement is excellent.

Summarizing, we have obtained a semiclassical approximation for the phase space representation of stationary states of spin systems. This was done by investigating the semiclassical Green function in the vicinity of real periodic trajectories. The accuracy of the result was verified by comparing it with exact calculations for the simple case $\hat{H}=\hbar \omega J_{z}$ and for the Lipkin-Meshkov-Glick model. Husimi functions are known to be good tools to study quantum chaos [13], and an extension of this theory to more degrees of freedom would be interesting in order to approach chaotic systems (a trace formula for chaotic spin systems was recently obtained [14] but, as already noted, taking the trace obliterates the information about the residues). Work in this direction is in progress.

We acknowledge financial support from Fapesp (Fundação de Amparo à Pesquisa do Estado de São Paulo).
[1] J. R. Klauder, Phys. Rev. D 19, 2349 (1979); G. J. Papadopoulos and J. T. Devreese, Path Integrals (Plenum, New York, 1978); J. R. Klauder and B.-S. Skagerstam, Coherent States: Applications in Physics and Mathematical Physics (World Scientific, Singapore, 1985).

[2] M. Baranger et al., J. Phys. A: Math. Gen. 34, 7227 (2001).

[3] S. Tomsovic and E. J. Heller, Phys. Rev. E 47, 282 (1993); A. L. Xavier Jr and M. A. M. de Aguiar, Ann. Phys. 252, 458 (1996); A. L. Xavier Jr and M. A. M. de Aguiar, Phys. Rev. A 54, 1808 (1996); A. L. Xavier Jr and M. A. M. de Aguiar, Phys. Rev. Lett. 79, 3323 (1997); F. Parisio and M. A. M. de Aguiar, Phys. Rev. A 68, 062112 (2003).

[4] T. VanVoorhis and E. J. Heller, Phys. Rev. A 66, 050501 (2002).

[5] A. D. Ribeiro, M. A. M. de Aguiar, and M. Baranger, Phys. Rev. E 69, 066204 (2004).

[6] D. Loss, D. P. DiVincenzo, and G. Grinstein, Phys. Rev. Lett. 69, 3232 (1992); J. von Delft and C. L. Henley, Phys. Rev. Lett. 69, 3236 (1992); A. Garg, Europhys.
Lett. 22, 205 (1993); A. Garg et al., J. Math. Phys. 44, 48 (2003).

[7] M. Stone, K.-S. Park, and A. Garg, J. Math. Phys. 41, 8025 (2000).

[8] H. G. Solari, J. Math. Phys. 28, 1097 (1987); E. Kochetov, J. Math. Phys. 36, 4667 (1995); V. R. Vieira and P. D. Sacramento, Nucl. Phys. B 448, 331 (1995).

[9] M. Pletyukhov, J. Math. Phys. 45, 1859 (2004).

[10] M. Brack and R. K. Bhaduri, Semiclassical Physics (Addison-Wesley, Reading, 1997).

[11] A. Garg and M. Stone, Phys. Rev. Lett. 92, 010401 (2004).

[12] J. Kurchan, P. Leboeuf, and M. Saraceno, Phys. Rev. A 40, 6800 (1989).

[13] P. Leboeuf and A. Voros, J. Phys. A: Math. Gen. 23, 1765 (1990); P. Leboeuf, J. Phys. A: Math. Gen. 24, 4575 (1991); T. Prosen, J. Phys. A: Math. Gen. 29, 5429 (1996); H. J. Korsch, C. Müller, and H. Wiescher, J. Phys. A: Math. Gen. 30, L677 (1997).

[14] M. Pletyukhov et al., Phys. Rev. Lett. 89, 116601 (2002). 